
allemande

49-1 | 2017

Berlin 1957-1994

\title{
La culture sous le sceau de la Guerre froide. Berlin
} 1957-1994

Boris Grésillon

\section{Q OpenEdition \\ 1 Journals}

Édition électronique

URL : https://journals.openedition.org/allemagne/517

DOI : 10.4000/allemagne.517

ISSN : 2605-7913

Éditeur

Société d'études allemandes

Édition imprimée

Date de publication : 16 juin 2017

Pagination : 23-35

ISSN : 0035-0974

\section{Référence électronique}

Boris Grésillon, « La culture sous le sceau de la Guerre froide. Berlin 1957-1994 », Revue d'Allemagne et des pays de langue allemande [En ligne], 49-1 | 2017, mis en ligne le 16 juin 2018, consulté le 01 juin 2022. URL : http://journals.openedition.org/allemagne/517 ; DOI : https://doi.org/10.4000/allemagne. 517 


\title{
La culture sous le sceau de la Guerre froide. Berlin 1957-1994
}

\author{
- Boris Grésillon*
}

\section{Introduction}

Berlin, 1957-1994. Pour le commun des mortels, les bornes chronologiques fixées par le jury de l'agrégation d'allemand n'évoquent pas grand-chose. Que ce soit pour ce qui est de l'histoire politique, géopolitique ou culturelle de Berlin, ces deux dates ne sont pas marquantes. Mais à y regarder de plus près, elles sont rattachées à deux événements qui, bien que n'étant pas de même nature, peuvent être malgré tout rapprochés.

1957 évoque avant tout l'Interbau, c'est-à-dire l'Internationale Bauausstellung, l'exposition internationale d'architecture de Berlin-Ouest, qui a marqué le paysage architectural de cette partie de la ville et qui a donné naissance au quartier de la Hanse, le Hansaviertel en bordure du Tiergarten. L'Interbau de Berlin-Ouest est une réponse politico-urbaine très claire à l'édification de la Stalinallee dans la partie Est de la ville, inaugurée en grande pompe en 1953 d'après les plans de l'architecte Hermann Henselmann. Autrement dit, la première borne chronologique fait clairement référence à l'histoire architecturale et urbanistique des deux Berlin.

Quant à 1994, on ne peut pas dire que ce soit une date marquante dans l'histoire architecturale, urbanistique et même culturelle de Berlin. Les très grands chantiers, ceux de la Potsdamer Platz et du quartier gouvernemental (Spreebogen, Reichstag, chancellerie, ministères), vont démarrer un peu plus tard, en 1995. De même, les mémoriaux (mémorial aux victimes de l'Holocauste, mémorial du Mur) et les musées mémoriels (musée juif construit par Daniel Libeskind, Topographie des Terrors) seront édifiés à la fin de la décennie 1990. En revanche, l'année 1994 revêt une certaine importance sur le plan politique car elle correspond au retrait définitif des troupes alliées de Berlin, autrement dit à la fin concrète de la Guerre froide sur le sol berlinois et allemand.

* Professeur de géographie à l'université d'Aix-Marseille, chercheur associé au Centre Marc Bloch et à I'Université Humboldt de Berlin. 
Du même coup, il est tentant d'interpréter le sujet proposé comme ressortant de l'histoire culturelle et politique de Berlin au temps de la Guerre froide. En essayant de trouver un lien entre un événement architectural en 1957 et un événement politique en 1994, le seul qui semble s'imposer est lié à la présence alliée dans Berlin. En effet, l'Interbau de 1957, on le verra, se déroule en partie sous l'influence de l'urbanisme états-unien. De plus, en 1957, les Américains inaugurent la Kongresshalle (aujourd'hui nommée Maison des cultures du monde, Haus der Kulturen der Welt), un cadeau qu'ils font à la ville de Berlin-Ouest, située non loin du Hansaviertel. De son côté, la Stalinallee, malgré ses quelques spécificités germaniques, correspond aux canons de l'art socialiste tels que dictés par Moscou. On peut donc considérer ces trois événements architecturaux comme des actes politiques. Et en 1994, ces mêmes Alliés, après avoir beaucoup investi le terrain berlinois, se retirent de la ville en laissant aux Berlinois le soin de décider de leur destinée. On peut raisonnablement en déduire que l'intention du jury est d'orienter les candidats vers une lecture politique, marquée par l'influence des Alliés, de l'évolution culturelle, artistique, architecturale et urbaine de Berlin entre 1957 et 1994. À l'appui de cette thèse, on rappellera que Berlin fut sous administration alliée pendant toute cette période et que sa marge de manœuvre politique, économique, culturelle fut singulièrement réduite.

Nous déclinerons cette perspective, à savoir «Berlin sous tutelle des Alliés», à travers trois périodes de durée inégale. Ainsi, on verra comment les deux Berlin se forgent une nouvelle identité dans la dépendance à l'égard des États-Unis d'un côté et de l'URSS de l'autre (années 1950 et 1960); puis comment dans les deux décennies suivantes (1970 et 1980), les deux villes s'émancipent progressivement de cette tutelle, sans qu'elle disparaisse pour autant; et enfin comment la chute du Mur et la réunification inaugurent une nouvelle ère marquée par l'effacement puis le retrait des forces alliées.

Dans un premier temps, entre 1957 et la fin des années 1960, Berlin est placé sous le signe de la reconstruction. Les deux décennies suivantes, 1970 et 1980, correspondent à la période des expérimentations. Enfin, la dernière période, 1990-1994, est placée sous le signe de la réunification et correspond à ce qu'on pourrait appeler l'apprentissage de l'autonomie.

\section{1957 - fin des années 1960 : la reconstruction dans la dépendance}

\section{En urbanisme et en architecture}

En 1957, Berlin se relève tout doucement de la guerre. Les habitants sont loin d'avoir effacé les stigmates des bombardements. Certains quartiers, à l'Est comme à l'Ouest, sont encore à moitié en ruines. La Kongresshalle va d'ailleurs être édifiée sur un champ de ruines. Elle figurera pendant longtemps comme un isolat au sein d'un no man's land en bordure du Tiergarten lui-même réduit à néant par les flammes pendant les bombardements alliés en 1945. Le bâtiment, contribution de l'architecte américain Hugh Stubbins à l'exposition internationale d'architecture, a été érigé en 1957. Cofinancé par le gouvernement américain, il a une valeur forte car il est censé symboliser l'amitié retrouvée et le dialogue intellectuel renoué entre les Allemands de l'Ouest et les Américains. On a beaucoup disserté sur son architecture inédite avec son toit en forme de coquillage ouvert. Ce qui est sûr, c'est que c'est un témoin précieux de ce qu'on pourrait qualifier $\mathrm{d}^{\prime}$ " architecture américaine de réconciliation ». 
Quant à l'Interbau, c'est bien plus qu'une vaste entreprise de reconstruction du Hansaviertel. C’est une réponse idéologique à la Stalinallee. De fait, tout les oppose. Autant la Stalinallee est une «magistrale» minérale impressionnante dans ses dimensions, construite sur le modèle des grands axes à la gloire du socialisme triomphant, autant le Hansaviertel est un ensemble d'immeubles variés disséminés dans la verdure du Tiergarten tout proche, construits par les plus grands architectes internationaux de l'époque (parmi lesquels Le Corbusier et Oskar Niemeyer). La Stalinallee est une avenue monumentale flanquée d'immeubles identiques. Le Hansaviertel est un quartier urbain dans la verdure au sein duquel chaque immeuble «signé» par un architecte de renom est différent. Ce qui est fascinant à travers ces deux réalisations, les premières vraiment marquantes de l'après-guerre, c'est qu'elles sont le fidèle reflet de deux idéologies, qui trouvent avec l'urbanisme et l'architecture un terrain privilégié pour s'exprimer $^{(1)}$. On voit bien à quel point les deux Berlin servent de vitrine à des grands desseins qui les dépassent. Plus que jamais, en pleine Guerre froide, l'architecture et l'urbanisme sont politiques.

\section{En art}

Il en va de même en art. La culture aussi est politique.

À Berlin-Est, les autorités se dépêchent de reconstruire ou de rénover les grandes institutions culturelles, comme le Staatsoper, le Deutsches Theater, l'île aux musées ou encore le Berliner Ensemble, qui accueillera la troupe de Bertolt Brecht de retour d'exil en 1949. Mais ce n'est pas tout. En RDA, comme dans toute République socialiste et plus généralement dans tout régime totalitaire, l'art est fortement teinté d'idéologie. Walter Ulbricht, le tout-puissant ministre-président de la RDA, cherche, dans les années 1950, à imposer un art officiel, qu'on a qualifié de «réalisme socialiste», à l'ensemble du milieu artistique. Devant la résistance d'une partie de celui-ci, le pouvoir change de tactique et impose en 1959 «la voie de Bitterfeld» (Bitterfelder Weg) qui consiste à inciter les ouvriers à s'emparer des outils de l'art pour exprimer le triomphe de la classe ouvrière - et à écarter les artistes «suspects» de ce même mouvement. Il s'ensuit une période de mise au pas de l'art et des artistes dissidents, qui culmine avec l'édification du Mur en 1961. La culture est-allemande vit alors ses heures les plus sombres. Mais paradoxalement, l'édification du Mur de Berlin va quelque peu desserrer l'étreinte du régime sur les artistes est-allemands. En effet, le Mur remplissant sa fonction essentielle, à savoir empêcher la fuite de dizaines de milliers de citoyens est-allemands à l'Ouest, l'État a beau jeu de se montrer un peu plus magnanime envers les artistes est-allemands, qui de toute façon sont désormais prisonniers en leur propre

1 Cela dit, hormis les projets de prestige qui concernent la partie centrale et que tout oppose, en périphérie, les grands programmes de logement se ressemblent davantage, avec de grands ensembles de tours et de barres comme il en fleurit partout en Europe dans les années 1960. Mais la spécificité du Berlin divisé, c'est de se construire dans l'opposition, afin de prouver au monde entier qu'on est supérieur à son frère ennemi. Ainsi, les grands ensembles de Marzahn puis de Hellersdorf à Berlin-Est répondent non seulement à un besoin impérieux de logements mais ils peuvent aussi être interprétés comme des réponses socialistes aux programmes de grands ensembles lancés à Berlin-Ouest au début des années 1960, avec le Märkisches Viertel au nord et Gropiusstadt au sud. Voir sur ce point la contribution de Hubert Guicharrousse dans ce numéro. 
pays et vont devoir tôt ou tard, pense le Politbüro du SED, rentrer dans le rang. Il résulte de cette fragile ouverture des ouvres critiques, qui marquent les années 1960 de leur empreinte, à l'instar du roman Der geteilte Himmel/Le ciel partagé de Christa Wolf (1963) et de son adaptation par le réalisateur Konrad Wolf (1964).

À Berlin-Ouest, la situation est fort différente, mais elle est également sur-déterminée par les champs de force de la géopolitique européenne. Ainsi, sur le plan culturel, il s'agit de rivaliser avec le riche paysage culturel de Berlin-Est. Par conséquent, grâce aux subventions de l'État fédéral et du plan Marshall, le Sénat de Berlin édifie un "Théâtre populaire libre» (Freie Volksbühne) pour répondre à la Volksbühne de l'Est comme on a édifié auparavant une université "libre» (Freie Universität) grâce aux fonds américains, en réponse à l'Université Humboldt située à Berlin-Est et jugée idéologiquement orientée. On construit, sur des plans de l'architecte Hans Scharoun, une deuxième bibliothèque nationale (Staatsbibliothek) pour faire pièce à son homologue de Berlin-Est. À proximité, toujours sur des plans de Scharoun, sera édifiée en 1963 la fameuse Philharmonie (2 400 places) ainsi que la Neue Nationalgalerie en 1968 par l'architecte Mies van der Rohe, le tout constituant un «forum culturel» («Kulturforum») devant concurrencer celui d'Unter den Linden à l'Est - qui comprend pour mémoire: le Staatsoper, le Komische Oper, le Maxim-Gorki-Theater, la Neue Wache, le Deutsches historisches Museum, le Berliner Dom, la Museumsinsel et ses cinq musées nationaux dont le Pergamon Museum, et un peu plus loin l'Admiralspalast, le Friedrichstadtpalast, le Berliner Ensemble et le Deutsches Theater.

Sur le plan radiophonique, la Guerre froide bat son plein. Dès 1946, pour contrer la radio mise en place par les Soviétiques, Américains et Britanniques créent une nouvelle radio, RIAS - Rundfunk im amerikanischen Sektor - qui va jouer un rôle essentiel auprès de la population de Berlin-Ouest jusqu'à la réunification. Adossés à la radio RIAS, un chœur (RIAS-Kammerchor), un orchestre symphonique (RSOB - RundfunkSymphonie-Orchester-Berlin) et d'autres formations musicales vont voir le jour dans les années 1950 pour faire pendant à leurs homologues de Berlin-Est (RundfunkOrchester und Chöre).

À la fin des années 1960, les deux dispositifs culturels de Berlin-Est et de BerlinOuest sont en place, et en observant cette ville qui voit double, au "ciel partagé», cette ville qui a désormais deux bibliothèques «nationales», deux galeries de peinture "nationales", deux chœurs et orchestres radiophoniques et deux Volksbühnen, on est comme pris de vertige, dans ce jeu de miroir incessant entre l'Est et l'Ouest et l'impression de paradis quelque peu artificiel qui en résulte. Les deux décennies suivantes vont proposer des paradis artificiels d'un tout autre ordre.

\section{Les décennies 1970-1980: les années d'expérimentation}

\section{1. À Berlin-Ouest}

Le mouvement étudiant marque évidemment une rupture dans l'histoire politique, sociale et culturelle de Berlin-Ouest comme dans celle des autres grandes villes d'Europe. À l'image de la ville et de sa jeunesse engagée politiquement, la révolution étudiante sera davantage violente que festive à Berlin-Ouest. La mort tragique de l'étudiant Benno Ohnesorg, abattu par un policier le 2 juin 1967 au cours d'une 
manifestation, puis l'attentat perpétré sur le leader étudiant Rudi Dutschke le 11 avril 1968 vont contribuer à durcir et à radicaliser le mouvement étudiant. Ne trouvant pas de réel débouché politique, hormis sous des formes extrêmes (cf. naissance de la fraction Armée rouge - Rote Armee Fraktion), il s'épanouira finalement sur le terrain artistique, culturel et social.

C'est la période de remise en question de l'ordre établi et de l'expérimentation sociale et artistique la plus radicale. Ainsi, des «Kommunen», c'est-à-dire des minicommunautés autogérées, des «WG» (pour Wohngemeinschaften), c'est-à-dire des appartements habités à plusieurs selon le principe de la colocation, se mettent en place, tandis que les squats illégaux fleurissent un peu partout dans le quartier de Kreuzberg essentiellement ${ }^{(2)}$, qui devient le symbole de la contre-culture en Allemagne de l'Ouest - ainsi que le symbole de la communauté turque, solidement implantée dans le quartier depuis l'arrivée des premiers «Gastarbeiter» dans les années 1960. Sur le plan artistique, la culture alternative autoproclamée expérimente des formes nouvelles, souvent à la frontière entre l'art et la politique: cirques itinérants, sit-ins, théâtre de rue, activisme à la façon du mouvement Dada, théâtre politique en langue turque (cf. le rayonnement du théâtre Tiyatrom de Kreuzberg, unique en son genre), nouveau théâtre pour enfants et adolescents (cf. succès du Grips Theater), cabarets politiques, cinéclubs critiques, etc.

À la fin des années 1970, un mouvement musical finit par s'imposer, sur le modèle de Londres, c'est le punk, avec des groupes légendaires comme PVC ou Nina Hagen Band, qui se produisent dans des lieux tout aussi légendaires, tels le club SO-36 ou le Tempodrom. L’attractivité de Kreuzberg sur la jeunesse rebelle de la RFA ne fait alors que grandir, renforcée par le fait que Berlin-Ouest est la seule ville d'Allemagne de l'Ouest où le service militaire n'est pas obligatoire. C'est ainsi que convergent vers Berlin-Ouest et notamment vers Kreuzberg tous les "Querdenker» de la nation (littéralement «ceux qui pensent de travers» ou «qui pensent autrement»), sur fond de consommation quasi légale de toutes sortes de drogues. Attirés par l'aura sulfureuse de Kreuzberg, des artistes internationaux comme David Bowie ou Iggy Pop passeront des années dans ce quartier, y créant quelques-uns de leurs meilleurs albums, et participant ainsi du mythe. Dans les années 1980, d'autres styles de musique s'imposent, plus électroniques, avec notamment Blixa Bargeld et les Einstürzende Neubauten (un nom imprononçable pour un groupe de légende).

Enfin, c'est aussi à Kreuzberg que naît un nouveau théâtre, la Schaubühne, dirigée par le metteur en scène Peter Stein et réunissant les meilleurs comédiens de cette génération, Jutta Lampe, Bruno Ganz, Edith Clever, Otto Sander. Ce théâtre organisé en «Kommune» où tout est partagé va également devenir un mythe, avant de s'exiler en 1981 sur le noble Kurfürstendamm.

Sur le plan architectural, l'heure est aussi à l'expérimentation. Dans le cadre des deux «IBA» (IBA-Alt et IBA-Neu) de l'Internationale Bauausstellung de 1984 à 1987, de nombreux îlots de Kreuzberg, Tiergarten ou Schöneberg sont reconstruits ou rénovés avec soin, comme l'expression de «rénovation douce» (behutsame Stadterneuerung), forgée pour l'occasion, le laisse entendre. Quant aux nouvelles constructions issues

2 Voir l'article de Patrick Farges sur les Hausbesetzer de Kreuzberg dans ce même numéro. 
de l'IBA-Neu, elles doivent respecter les principes de la «reconstruction critique» (kritische Rekonstruktion) édictés par l'architecte Josef-Paul Kleihues, qui imposent aux maîtres d'œuvre de respecter les dimensions traditionnelles (hauteur, longueur) des immeubles berlinois. Ainsi, au grand dam des promoteurs, les constructions en hauteur, pourtant à la mode au début des années 1980, sont bannies. Il faut ici souligner le rôle majeur des «activistes» de Kreuzberg, qui, dès les années 1970, s'opposent farouchement à la destruction façon table rase (Kahlschlagsanierung) de très nombreux immeubles «Gründerzeit» (construits au tournant des XIX ${ }^{\mathrm{e}}$ et XX $\mathrm{X}^{\mathrm{e}}$ siècles), certes endommagés par la guerre mais susceptibles d'être réhabilités. D’une certaine manière, l'IBA vient couronner leurs succès, arrachés de haute lutte à travers des actions spectaculaires d'occupation des immeubles. Comme le dit avec du recul Hardt-Waltherr Hämer, le directeur de l'IBA-Alt:

"À l'époque, la rénovation urbaine était un acte révolutionnaire contre le cartel de l'économie immobilière et de la politique. Aujourd'hui elle est pratiquée quotidiennement dans la ville existante» ${ }^{(3)}$.

Ce que la rénovation urbaine à Kreuzberg comportait, entre autres, de révolutionnaire, c'est qu'elle impliquait systématiquement les habitants dans la rénovation de leurs logements. Ces derniers étaient les interlocuteurs de l'architecte et avaient le pouvoir de faire des suggestions. Ce processus de construction démocratique a, certes, un peu ralenti l'édification des immeubles mais il s'en est ensuivi des constructions variées, originales, de grande qualité, «öko» avant l'heure, visibles par exemple le long du Landwehrkanal. S’agissant essentiellement de logements sociaux, destinés aussi bien à des familles nombreuses d'origine turque qu'à des couples sans travail, on peut dire qu'une certaine forme d'utopie était devenue réalité.

Plus généralement, il est frappant de constater qu'en l'espace de deux décennies, dans la foulée de mai 1968, les acteurs alternatifs de Berlin-Ouest vont inventer de nouveaux genres artistiques, de nouvelles façons d'habiter et de faire la ville qui sont révolutionnaires pour l'époque et qui, pour certains, sont rentrés dans les mœurs depuis. Ce qui caractérise la période, c'est à la fois le goût du risque, l'inventivité et une conscience politique aiguë de la part d'acteurs culturels militants. Les résultats de ce cocktail détonnant frappent par leur audace et leur avant-gardisme. Le quartier de Kreuzberg en fut le principal réceptacle, et secondairement les arrondissements voisins de Schöneberg, Tiergarten et Tempelhof.

\section{2. À Berlin-Est}

Côté Est aussi, les choses bougent, même si c'est moins visible, moins spectaculaire qu'à Kreuzberg. N'oublions pas qu'en régime totalitaire, l'art et la culture ont à se conformer à l'idéologie promue par le pouvoir, en l'occurrence celle du « réalisme socialiste». Les artistes qui dérogent à la règle sont catalogués comme «anti-communistes », «anti-socialistes», «ennemis du peuple», «occidentaux» et sont rapidement

3 «Damals war Stadterneuerung ein revolutionärer Akt gegen das Machtkartell von Wohnungswirtschaft und Politik. Heute ist sie zum alltäglichen Umgang mit der bestehenden Stadt geworden.» Source: site de la Société d'architecture d'Autriche, ÖGFA, consulté le 16.02.2017, https://oegfa.at/ programm-1/vortraege/behutsame-stadterneuerung-und-iba-alt. 
interdits de publication ou de représentation. Combien d'architectes non conformes n'ont jamais pu construire un bâtiment! Combien d'écrivains ont vu leur espoir de publication anéanti par la censure! Combien de metteurs en scène dissidents n'ont jamais pu franchir les portes d'un théâtre? On ne le saura jamais. Néanmoins, à partir de 1970, une forme de culture alternative commence à naître; elle porte les noms évocateurs de «culture de niche» (Nischenkultur) ou de «sub-culture» (Subkultur). Elle ne peut en effet prétendre à plus. Mais elle est bien présente dans les interstices du pouvoir et de la culture officielle.

Dans toutes les grandes villes est-allemandes, Leipzig, Dresde, Halle, Erfurt, Berlin-Est, se met en place une «culture de niche». Celle-ci prend la forme de galeries autonomes, de cercles littéraires, d'ateliers qui se développent dans des arrière-cours ou des appartements privés, en tout cas loin des regards de la Stasi (en fait on sait aujourd'hui que la «Szene» artistique est-allemande faisait l'objet d'une surveillance étroite et même d'une infiltration quasi-systématique par des "agents informateurs» de la police politique). Ainsi, en littérature, des auteurs majeurs comme Christa Wolf, Heiner Müller, Volker Braun ont maille à partir avec le SED mais parviennent quand même, grâce à leur notoriété, à publier leurs romans, tandis que l'écrivain opposant Stefan Heym se fera publier en RFA tout en restant fidèle à la RDA. Il est à noter que ces écrivains de Berlin-Est, éminemment respectés et lus dans les deux Allemagne, ont largement contribué, par leurs écrits puis par leurs actions concrètes lors des derniers mois de la RDA, à saper l'édifice du Mur.

En peinture, on constate également que les grands peintres est-allemands, tels ceux de "l'école de Leipzig", bousculent les canons de l'art pictural des deux côtés du rideau de fer. Ainsi, lors de la $6^{\mathrm{e}}$ Documenta de Kassel en 1977, les organisateurs réservèrent pour la première fois une section du Salon à des représentants du « réalisme socialiste» de RDA. Les peintres de «l'école de Leipzig», Bernhard Heisig, Wolfgang Mattheuer, Willi Sitte et Werner Tübke, d'authentiques créateurs interprétant à leur façon (critique, abstraite, faussement historicisante, etc.) la ligne officielle du « réalisme socialiste", firent sensation et déclenchèrent une vive polémique auprès de leurs confrères ouest-allemands, dont certains se retirèrent du Salon en signe de protestation, tandis que d'autres, ainsi que des collectionneurs notamment, manifestèrent de l'intérêt pour leurs œuvres.

De la même manière, on peut prendre l'exemple du cinéma est-allemand. Derrière les comédies médiocres ou les films officiels sans importance, quelques chefs-d'œuvre ont pu voir le jour. Parmi ceux-ci, il faut citer le film Die Legende von Paul und Paula de Heiner Carow (La légende de Paul et Paula) qui fut autorisé à sortir sur les écrans en 1973 grâce à l'intervention de Erich Honecker en personne, qui y voyait un film d'amour destiné à la jeunesse. Certes, mais Paul und Paula est avant tout une ode à la liberté, à l'amour passion et à la poésie. Ce film «libertaire» filmé à Berlin-Est comporte donc un aspect subversif évident. Le public est-allemand, qui s'est précipité dans les salles dès sa sortie, ne s'y est pas trompé. Dans un tout autre registre, on peut également citer le film Die Architekten de Peter Kahane, qui sort sur les écrans en mai 1990 mais dont le scénario avait été autorisé par le régime en 1989, juste avant la chute du Mur. Ce film, qui conte les déboires d'un jeune collectif d'architectes de Berlin-Est broyé et interdit de construire par les fonctionnaires du ministère, a valeur 
de symbole pour toutes les tentatives créatives de réformer le système de l'intérieur. Comme Die Legende von Paul und Paula, Die Architekten est tourné à Berlin-Est, confirmant un peu plus le statut spécial de la capitale de la RDA.

Contrairement à ce que l'on pourrait penser, les relations entre artistes de BerlinEst et de Berlin-Ouest, plus généralement entre créateurs de RDA et de RFA, étaient réelles et parfois régulières. Il s'agissait, certes, de se jauger, mais aussi d'apprendre à se connaître. Ainsi, on sait aujourd'hui que les architectes et urbanistes des deux Berlin entretenaient des liens secrets, du moins très discrets pour échapper à la surveillance du régime est-allemand. Même l'architecte officiel du régime et bâtisseur de la Stalinallee Hermann Henselmann rencontrait parfois ses confrères de BerlinOuest et échangeait librement avec eux. Cet exemple jette une autre lumière sur les rapports artistiques entre l'Est et l'Ouest sous la Guerre froide, qui étaient bien plus complexes qu'on l'a longtemps pensé et qui ne ressortaient pas de la seule confrontation idéologique directe ou de l'ignorance de l'autre. Pour reprendre l'exemple du film Die Legende von Paul und Paula, il fut montré en RFA avec le même succès qu'en RDA puis passa ensuite pendant des années sur les chaînes de télévision publique. Les deux acteurs principaux ont ainsi acquis une certaine notoriété à l'Ouest, ce qui facilita sans doute leur passage en Allemagne de l'Ouest au début des années 1980.

Au niveau de la géographie artistique de la RDA, Berlin-Est, de par son statut de capitale d'un État centralisé, revêt à la fin de la période tous les atours d'une capitale culturelle. Même si les deux capitales saxonnes, Leipzig (avec "l'école de Leipzig», l'école des beaux-arts, l'orchestre du Gewandhaus) et Dresde (avec l'opéra du Semperoper, les musées royaux - «Zwinger»), constituent d'incontestables pôles culturels majeurs, Berlin-Est concentre malgré tout les studios de cinéma de la DEFA à Babelsberg, les joyaux de l'île aux musées, une scène théâtrale et orchestrale très dense, deux opéras, une bibliothèque nationale, auxquels vient s'ajouter en 1984 à Berlin-Mitte le Friedrichstadt-Palast, «le plus grand et le plus moderne théâtre de divertissement d'Europe» (ce qu'il est encore aujourd'hui).

$\mathrm{Au}$ niveau de la scène alternative, le pendant de Kreuzberg à Berlin-Est, toutes choses égales par ailleurs, est le quartier de Prenzlauer Berg, qui fait figure de quartier de la bohème et de la contestation en RDA. C'est là que se réunissent de nombreux artistes, écrivains, architectes dissidents. C'est de là aussi qu'un certain nombre d'entre eux choisiront l'exil à l'Ouest après "l'affaire Biermann ", autrement dit après l'expulsion en novembre 1976 du chanteur hostile au régime, Wolf Biermann. Parmi eux, les deux acteurs principaux de Paul und Paula, Angelica Domröse et Winfried Glatzeder, expulsés en 1981.

À l'issue de ce parcours à travers les années 1970 et 1980 dans les deux Berlin, une question se pose: comment expliquer cette soudaine floraison, de part et d'autre du Mur, d'une culture contestataire alors même que les deux moitiés de ville continuent de vivre sous tutelle alliée? C’est justement parce qu'au début de la décennie 1970, la situation politique évolue. Avec l'accord quadripartite de 1971, Berlin-Ouest est reconnu officiellement et acquiert davantage d'autonomie par rapport aux Alliés américains, britanniques et français. En outre, la démographie en berne de BerlinOuest pousse les autorités sénatoriales et fédérales à inciter les jeunes Allemands de 
l'Ouest à s'établir à Berlin-Ouest, en passant sous silence le fait qu'un grand nombre d'objecteurs de conscience, d'étudiants engagés politiquement et de "Querdenker» se trouvaient parmi eux. Le terreau subversif de Kreuzberg s'en trouva singulièrement renforcé.

Quant à la RDA, le passage de témoin entre Walter Ulbricht et Erich Honecker à la tête du Comité central du SED en 1971 laisse dans un premier temps espérer une tolérance accrue du pouvoir vis-à-vis des créateurs. Effectivement, la sortie du film Paul und Paula en 1973 viendrait corroborer cette thèse. En fait, le dégel fut de courte durée et l'expulsion de Wolf Biermann en 1976 marque clairement une reprise en main du pouvoir.

Côté Ouest, les utopies des années 1970 finissent par s'épuiser à la fin des années 1980. Enfin, l'année 1987 marque l'apogée de la rivalité idéologico-culturelle entre les deux moitiés de ville, à l'occasion du $750^{\mathrm{e}}$ anniversaire de la ville, fêté en grande pompe des deux côtés du Mur ${ }^{(4)}$. À Berlin-Est, le Schauspielhaus de l'architecte Karl Friedrich Schinkel est rouvert au public après des années de restauration. Berlin-Ouest «riposte» avec l'inauguration de la Kammermusiksaal, la salle de concert voisine de la Philharmonie. D'un côté, on rouvre une salle de concert traditionnelle édifiée en 1821; de l'autre, on inaugure une salle de concert ultra-moderne. À Berlin-Est, on organise parades et inaugurations officielles. À Berlin-Ouest, on préfère lancer de nouveaux festivals et inviter des artistes du monde entier à célébrer la "vitrine de l'Occident». Sur le plan urbanistique et architectural, Berlin-Ouest fête le succès de l'IBA et de son concept de "rénovation douce», qui préserve le bâti existant; les autorités de BerlinEst se félicitent quant à elles de l'avancée du tout nouveau chantier lancé un an plus tôt, la «ville-satellite» de Hellersdorf constituée de barres et située à l'extrême est de la ville. Plus que jamais, les deux villes se tournent le dos, font semblant de s'ignorer alors qu'elles ne cessent de s'épier, et se développent consciemment l'une contre l'autre. Mais dans les deux cas, à la fin de la décennie 1980, les utopies sont à bout de souffle, les modèles idéologiques s'épuisent, comme s'ils tournaient à vide.

\section{1989-1994: le début d'une nouvelle ère}

Nous ne revenons pas sur les événements liés à la chute du Mur le 9 novembre 1989, sauf pour signaler quand même que les artistes dissidents de l'Est dont nous avons parlé, tous ceux qui ont été muselés si longtemps par le pouvoir, ont joué un rôle important dans sa chute. Dans les manifestations de Leipzig ou de Berlin, ils ont souvent été aux premières loges, même si l'Histoire les a oubliés ensuite.

La chute du Mur de Berlin, totalement inattendue à l'Est comme à l'Ouest, inaugure pour la ville une période inédite à plus d'un titre.

\section{La nouvelle donne géopolitique...}

D’une part, sur le plan politique qui nous intéresse, autrement dit du point de vue des Alliés, le traité dit « $4+2 »$ puis la réunification allemande le 3 octobre 1990 scellent la fin de l'influence des quatre puissances alliées sur l'Allemagne et sur Berlin. Entre

4 Voir l'article de Krijn Thijs dans ce même numéro. 
l'effacement de l'armée soviétique et la débandade généralisée du Bureau politique du SED et du Comité central, les Allemands de l'Est se trouvent bienheureux mais orphelins d'une direction politique et les Berlinois de l'Est sans personne à leur tête. Côté Ouest, la situation est nettement moins chaotique, d'autant plus que Helmut Kohl est réélu chancelier triomphalement fin 1990 par les deux Allemagne. Mais à BerlinOuest, les Alliés ne pèsent plus et le premier maire élu de la ville réunifiée, Walter Momper, a bien d'autres soucis que de s'occuper de culture. Enfin, Berlin redevient officiellement capitale de l'Allemagne en juin 1991 mais il faudra attendre la toute fin de la décennie 1990 pour que les institutions fédérales s'installent réellement sur les bords de la Spree. Autrement dit, les années 1990 constituent pour Berlin une période de latence politique, dont les acteurs culturels vont profiter.

\section{2. ... et ses conséquences sur le terrain}

Le retrait des troupes alliées a des conséquences à court et moyen terme sur la géographie culturelle du Berlin réunifié. Comme on pouvait s'y attendre, les studios de cinéma de la DEFA à Babelsberg furent repris et continuent de fonctionner comme tels aujourd'hui. De même, l'immeuble de la radio RIAS abrite aujourd'hui Deutschlandradio ainsi que d'autres radios. Même si la plupart des sites militaires, comme le «quartier Napoléon», base de la principale garnison française située dans le quartier de Reinickendorf, furent rétrocédés à la Bundeswehr qui y installa ses troupes, d'autres connurent des fortunes plus inattendues. Ainsi, l'aéroport militaire américain de Tempelhof fonctionna d'abord quelques années comme aéroport civil, puis fut fermé à la fin des années 1990 du fait de sa trop grande proximité avec les habitations. Après une longue période de stase, il trouva une fonction culturelle, ses vastes hangars désaffectés accueillant des concerts de musique techno en été. Puis ce fut la partie extérieure qui fut spontanément utilisée en 2010 par la population sans que le Sénat de Berlin ne s'y oppose. Le site immense devint rapidement un espace de loisirs, de sport et de culture (cirque, théâtre en plein air, jonglerie...) approprié par les Berlinois, ce qu'il est plus que jamais après le succès du référendum de 2014 qui «interdit» au Sénat d'y envisager quelque construction que ce soit. Enfin, depuis l'arrivée massive de réfugiés syriens, irakiens, afghans à Berlin à partir de l'été 2015, l'aéroport lui-même et ses hangars ont servi et servent encore de lieu d'accueil et d'hébergement provisoire pour les réfugiés. Quelle destinée pour cet aéroport édifié par les nazis dans les années 1930, épargné par les bombardements alliés en 1945, transformé en 1948 en base du pont aérien qui permit de sauver Berlin-Ouest du blocus soviétique, utilisé tel quel par l'armée américaine pendant 40 ans, fermé à la fin des années 1990, rendu aux Berlinois dans les années 2010 puis utilisé depuis 2015 pour l'accueil de dizaines de milliers de réfugiés ! Tempelhof est à lui seul un condensé de la géopolitique mondiale depuis 80 ans.

On pourrait en dire autant de la station d'espionnage américaine installée au sommet de la colline du Teufelsberg (la «montagne du diable»), dans la forêt de Grunewald. À l'origine, il y avait à cet emplacement une université militaire conçue par l'architecte en chef Albert Speer, très solidement ancrée dans le sol. Les Alliés ne parvenant pas à la détruire, ils décidèrent de la recouvrir de milliers de tonnes de gravats issus des bombardements. Du haut de cette colline artificielle, les Américains installèrent une puissante station d'espionnage depuis laquelle ils captaient les signaux hertziens en provenance du bloc de l'Est. Après la chute du Mur, le matériel fut évacué par les Américains et la 
station fut fermée. Dans les années 1990, des investisseurs firent construire des hôtels et des petits immeubles mais le projet n'aboutit pas. C'est là que la culture reprit ses droits. Pendant quelques années, le Teufelsberg fut squatté par une colonie d'artistes qui entreprit de réhabiliter le lieu. La «colline aux grandes oreilles» devint ainsi un lieu d'expérimentation artistique et social. Aujourd'hui, les autorités ont mis fin à cette occupation illégale mais en autorisent l'accès pour des visites guidées. Lieu de formation pour les jeunes nazis, montagne de gravats après la Deuxième Guerre mondiale, principale station d'espionnage des Anglais et des Américains pendant la Guerre froide, fermeture puis renaissance avortée en tant que gros projet immobilier dans les années 1990, utopie sociale et culturelle temporaire, enfin tentative fragile de mise en tourisme: à sa façon, le Teufelsberg résume bien l'incroyable destinée de Berlin.

Pour revenir à notre propos, le départ des Alliés en 1994 a libéré énormément de foncier partout dans la ville et a parfois permis à des projets culturels inattendus de voir le jour. Entre le retrait des forces alliées et l'absence des organes fédéraux sur les bords de la Spree jusqu'en 1999-2000, les acteurs culturels se sont engouffrés dans la brèche et ont, au sens propre, occupé le terrain.

\section{La culture et les artistes à l'honneur}

Profitant consciemment ou inconsciemment de ce vide politique absolument inédit dans l'histoire de Berlin, les artistes, écrivains, créateurs, mais aussi responsables de structures artistiques, médiateurs culturels, opérateurs culturels, investissent le terrain berlinois et font très vite parler d'eux.

Que ce soit les artistes allemands connus comme Frank Castorf à la tête de la Volksbühne depuis 1992 ou Thomas Langhoff nommé directeur du Deutsches Theater en 1991, ou les artistes étrangers qui n’hésitent pas une seconde à reprendre les rênes de prestigieuses institutions berlinoises, tels Claudio Abbado à la direction de l'orchestre philharmonique de Berlin, Daniel Barenboim à la tête de l'orchestre du Staatsoper d'Unter den Linden ou Vladimir Ashkenazy à la direction du DSO (Deutsches Symphonie-Orchester), tous ces créateurs, au firmament de leur carrière, vont marquer de leur empreinte le paysage musical et culturel post-chute du Mur, et on mesure mieux aujourd'hui à quel point ils ont constitué une chance inestimable pour le renouveau culturel de la ville.

Mais Berlin ne saurait se résumer à ses institutions de prestige. La scène off, après les expérimentations des années 1970, va connaître un nouvel âge d'or dans les années 1990. La chute du Mur libère littéralement des énergies créatrices trop longtemps muselées, et en l'absence de pouvoir politique visible, les esprits rebelles de l'Est comme de l'Ouest prennent d'assaut la ville, occupant illégalement d'anciennes friches (cf. Tacheles), transformant des usines ou des entrepôts désaffectés en clubs technos (cf. Bunker, E-Werk, Tresor), squattant chaque appartement inoccupé des quartiers de Mitte et de Prenzlauer Berg. Et il y en avait beaucoup, à cause du départ précipité de nombreux citoyens est-allemands profitant des premières brèches dans le rideau de fer (cf. passages possibles à l'Ouest via la frontière entre la Hongrie et l'Autriche à l'été 1989) ou tournant le dos à la RDA dès les premières heures de l'ouverture du Mur.

C'est une période magique, où tout est possible. Le centre-ville de Berlin devient une sorte de scène à ciel ouvert où on refait le monde, même si les tensions restent 
vives entre artistes de l'Est et artistes de l'Ouest. Les artistes étrangers, venant des quatre coins du monde, ne sont pas en reste, trouvant en Berlin ces espaces de création dont ils rêvaient, contribuant aussi à la réputation de la ville à l'étranger. La géographie culturelle de la métropole retrouvée se redessine. Les arrondissements de Mitte et Prenzlauer Berg, centraux et aux loyers très abordables, deviennent in, Kreuzberg devient out. Pour la première fois dans l'histoire des capitales européennes, l'une d'entre elles, Berlin, devenait en son centre un terrain de jeu pour les créateurs du monde entier. C'est en cela que Berlin est unique et que les années 1990 constituent un moment exceptionnel de l'histoire de la ville.

\section{Conclusion}

Cette période a fini, comme toutes les utopies, par s'achever. En gros, elle a duré dix ans, éventuellement divisible en deux, les cinq premières années, de 1989 à 1994, étant celles de tous les possibles, et les cinq dernières années, de 1994 à 1999, la période de l'affirmation du off et d'un début d'institutionnalisation. En 1999-2000, avec l'installation des organes fédéraux à Berlin, avec l'achèvement des immenses chantiers de la Potsdamer Platz et de la Friedrichstraße, dévolus au commerce et au divertissement, avec l'irrépressible essor du tourisme, avec enfin le début d'un processus de gentrification marqué à Mitte et Prenzlauer Berg, Berlin change d'époque en changeant de siècle. La double reprise en main politique, par le pouvoir municipal et par le pouvoir fédéral, se marque par leur volonté que les choses rentrent dans l'ordre, que la ville se normalise après avoir été si longtemps «anormale», que les aspérités berlinoises se lissent, et aussi que la culture devienne «sexy»... et rentable. L'expression «Arm, aber sexy» ("pauvre mais sexy») lancée par le maire de Berlin Klaus Wowereit en 2003 devient le slogan officiel de la ville tout au long des années 2000. On peut se demander s'il n'est pas remplacé dans la décennie 2010 par l'expression "Reich und sexy geht auch» («riche et sexy, ça marche aussi») tant l'argent s'affiche dans les quartiers désormais totalement gentrifiés de Mitte, Prenzlauer Berg et Friedrichshain - ceux-là même, cruelle ironie de l'histoire, qui avaient été le berceau des squats et de la contreculture 25 ans auparavant.

Au demeurant, le moteur créatif berlinois ne s'est pas éteint, loin s'en faut. Côté pile ou officiel, les pionniers des grandes institutions berlinoises ont trouvé leurs dignes héritiers, qui se nomment Simon Rattle à la tête du Philharmonique, Thomas Ostermeier à la direction de la Schaubühne ou la chorégraphe Sasha Waltz à la tête du Radialsystem. Côté face ou alternatif, il est vrai que la plupart des squats d'artistes ont disparu et que la scène off n'a plus rien à voir avec celle de la décennie 1990, mais elle est toujours présente dans tous les domaines de l'art et de l'architecture, suscitant toujours l'attention des professionnels et du public. Les mythes ne meurent jamais complètement.

\section{Indications bibliographiques}

Volker Braun, Training des aufrechten Gangs. Gedichte, Halle, Mitteldeutscher Verlag, 1979.

Cyril Buffet, Berlin, Paris, Fayard, 1993.

Collectif, Oper Dynamo West - Die Stadt als Bühne, Ostfildern, Hatje Cantz Verlag, 2010.

Claire Соцомв, Staging the new Berlin, Londres/New York, Routledge, 2012. 
Boris GrésIllon, Berlin métropole culturelle, Paris, Belin, 2002.

Boris Grésillon, «Le Tacheles, histoire d'un 'SquArt' berlinois», Multitudes, 17 (2004), p. 147157.

Boris GrésILlon, "Contre-culture, musique et urbanisme: le cas emblématique de BerlinKreuzberg, de la fin des années 1960 à aujourd'hui», in: Charlotte Bomy, André ComBes, Hilda Inderwildi (dir.), Contre-cultures à Berlin des années 1960 à nos jours, Cahiers d'études germaniques, 64 (2013).

Ares Kalandides, «Berlin: les industries créatives et l'aménagement urbain», in: J.-J. Terrin (dir.), La ville des créateurs, Marseille, Parenthèses, 2012, p. 30-49.

Hélène Miard-Delacroix, Le défi européen. Histoire franco-allemande de 1963 à nos jours, Lille/Villeneuve d'Ascq, Presses du Septentrion, 2011.

Anaïs Prosaïc, «Rockin’ Berlin », Autrement, numéro spécial sur «Berlin, le ciel partagé», Paris, janvier 1983, p. 120-128.

Tobias Rapp, Lost and Sound. Berlin, Techno und den Easyjet Set, Francfort-sur-le-Main, Suhrkamp/Insel, 2009.

Régine RoBin, Berlin chantiers, Paris, Stock, 2001.

David Sanson (dir.), Berlin, Paris, Robert Laffont, 2015.

Wim Wenders, Les ailes du désir, 1987.

Christa Wolf, Der geteilte Himmel, Halle, Mitteldeutscher Verlag, 1963.

\section{Résumé}

Entre 1957 et 1994, c'est-à-dire entre l'inauguration de l'exposition internationale d'architecture de Berlin-Ouest et le retrait des troupes alliées de Berlin, la ville est profondément marquée par la Guerre froide et l'occupation des quatre puissances victorieuses. Celles-ci imposent leurs normes architecturales, urbaines et culturelles. On peut diviser la période en trois séquences: de 1957 à 1969, le maître-mot, à Berlin-Ouest comme à Berlin-Est, est la reconstruction sous contrôle allié; les deux décennies suivantes, de 1970 à 1989, sont celles de toutes les expérimentations; enfin, la chute du Mur fin 1989 inaugure une ère nouvelle, où le Berlin réunifié doit réapprendre à ne faire qu'un, sans la tutelle alliée. Au total, cette séquence de 40 ans aura été tout sauf une parenthèse historique. Malgré la division et les déchirements, ce fut une période fondatrice.

\section{Zusammenfassung}

Zwischen 1957 und 1994, d.h. zwischen der Eröffnung der Internationalen Bau-Ausstellung (IBA) in West-Berlin und dem Rückzug der Allierten, ist die Stadt vom Kalten Krieg und von der Besatzung der vier Siegesmächte tief geprägt. Diese setzen ihre architektonischen, städtebaulichen und kulturellen Normen durch. Die Gesamtperiode lässt sich in drei Teile einteilen: Zwischen 1957 und 1969, in West-Berlin wie in Ost-Berlin, heißt das Schlüsselwort Rekonstruktion unter allierte Kontrolle; die nächsten zwei Jahrzehnte, von 1970 bis 1989, sind von vielen Experimenten geprägt; schließlich beginnt mit dem Mauerfall Ende 1989 eine neue Ära, in der das wiedervereinigte Berlin wieder lernen muss, zusammenzuwachsen, und das ohne den Einfluss der Allierten. 